\title{
Neuronavigation-guided endoscopy for intraventricular tumors in adult patients without hydrocephalus
}

\author{
Krzysztof Stachura, Ewelina Grzywna \\ Department of Neurosurgery and Neurotraumatology, Jagiellonian University, Medical College, Krakow, Poland
}

Videosurgery Miniinv 2016; 11 (3): 200-207

DOI: $10.5114 /$ wiitm.2016.61430

\begin{abstract}
Introduction: Intraventricular endoscopic operations are usually undertaken in patients with an enlarged ventricular system that provides good access to the ventricles, proper anatomic orientation and safety of maneuvers within the ventricles.

Aim: The preliminary assessment of the feasibility of endoscopic procedures in cases occurring without hydrocephalus. Material and methods: Eleven patients with intraventricular tumor diagnosed in neuroimaging studies were included in the study. None of these cases was accompanied by hydrocephalus. Surgery was performed with a rigid neuroendoscope using a neuronavigation system. The purpose of the operation was tumor removal or histological verification.

Results: The colloid cyst of the third ventricle was removed in 5 patients. In 1 patient a glial-derived tumor adjacent to the interventricular foramen was partially resected. In 1 case a tumor of the lateral ventricle was totally removed, and in another case the resection of such a tumor was partial. In 2 cases, a biopsy of the tumor of the posterior portion of the third ventricle was undertaken, while in 1 case the biopsy was abandoned due to the risk of injury of structures surrounding interventricular foramen. There were no intraoperative or postoperative complications. None of the patients developed hydrocephalus in the long-term follow-up. The results of treatment in the study group did not differ from those obtained in patients operated on with hydrocephalus.

Conclusions: The presence of hydrocephalus is not necessary to perform endoscopic surgery. However, in each case it should be preceded by a thorough analysis of the feasibility of the endoscopic procedure and should be supported by a neuronavigation system.
\end{abstract}

Key words: neuroendoscopy, neuronavigation, intraventricular tumors, narrow ventricular system.

\section{Introduction}

Intraventricular endoscopic surgery usually is undertaken in patients with an enlarged ventricular system. This provides good access to the ventricles, the proper anatomic orientation and the latitude of maneuvers within the ventricles. Grunert et al. postulated that patients with hydrocephalus resulting from aqueductal stenosis are the best candidates for endoscopic treatment [1]. In other cases, the effec- tiveness of this procedure might be limited [2]. The progress of endoscopic techniques, the new methods of imaging and the development of stereotactic systems seem to open new possibilities to conduct endoscopic procedures, even in patients without hydrocephalus [3-7]. The modern frameless neuronavigation systems refer the location of tools in the surgical field to the preoperative - reference - imaging study. They have allowed for precise planning of surgery, and the surgeon's hand during the proce-

\section{Address for correspondence}

Krzysztof Stachura MD, PhD, Department of Neurosurgery and Neurotraumatology, Jagiellonian University, Medical College, 3 Botaniczna St, 31-503 Krakow, Poland, phone: +48 1242486 60, e-mail: kkstach@poczta.onet.pl 
dure has been led starting from the ventricle cannulation to the accomplishment of the surgical target. This allows for the reduction of surgical injury to the intra- and periventricular structures $[4,5]$.

\section{Aim}

The aim of this study was to evaluate the endoscopic treatment results in patients with a ventricular system tumor without concomitant active hydrocephalus.

\section{Material and methods}

Eleven patients treated within 5 years were included in the study and followed with prospective observation. Intraventricular tumor was diagnosed based on the neuroimaging study with contrast-enhanced head computed tomography (CT) or magnetic resonance imaging (MRI) or both in all patients. In 6 patients the tumor was located in the anterior part of the third ventricle, in 3 patients in the posterior part of the third ventricle, and in the remaining 2 patients in the lateral ventricle. None of these cases was accompanied by hydrocephalus. The study group consisted of 7 women and 4 men aged 23-68 years (mean: 43 years). The symptomatology observed in patients prior to hospitalization varied. Headache was predominant in 6 cases, 5 patients suffered from short-term qualitative and quantitative impairments of consciousness, and 2 patients experienced visual disturbances. In 3 cases the tumor did not result in any neurological symptoms and was diagnosed accidentally in the head CT scan performed due to the head injury. Duration of symptoms ranged from $24 \mathrm{~h}$ to 9 months. In 4 cases it was difficult to determine.

All neuroimaging studies were evaluated by the same team of radiologists. To avoid overdiagnosis of hydrocephalus the frontal and occipital horn ratio was calculated. It has high reliability independent of age and correlates well with volumetric measurements. The ratio of 0.37 (with a standard deviation of 0.026 ) has been recognized as normative [8]. The size of tumors qualified for surgical treatment did not exceed $2-3 \mathrm{~cm}$ in the greatest dimension. Neuroimaging studies did not demonstrate them to be richly vascularized or to be of increased cohesion.

The purpose of the endoscopic procedure was tumor removal or histological verification. Furthermore, in patients with tumors located in the poste- rior part of the third ventricle third ventriculostomy was planned additionally. Prior to the surgery a thin layer (1-2 mm) head CT scan - reference study was performed in all patients. It was loaded into the Medtronic S7 Stealth Station (planning station of neuronavigation system). All patients were operated on under general anesthesia. During the procedure the patient's head was fixed to the operation table using a Mayfield clamp. Nine patients were operated on in the supine position with the head elevated to approximately $30^{\circ}$. In 2 cases of lateral ventricle tumors located near the occipital horn the patients were lying on their side with their head rotated in the direction opposite to the operation side. Based on the reference study an image of three basic planes (axial, sagittal, coronal) and a three-dimensional model were created. This allowed the burr hole location to be planned and the optimal access to the surgical target to be determined. The optical navigation enabled the spatial model of the navigation area to be built after registration of the patient position in relation to the planning station. This allowed for constant real-time surgical field tracking and its changes. Surgical instruments registered into the neuronavigation system were the standard ventricular drain and the endoscope sheath. The rigid Karl Storz neuroendoscopes were used: the GAAB model with Hopkins $0^{\circ}$ optics (having the outer diameter of $6.5 \mathrm{~mm}$ and $3 \mathrm{~mm}$ working channel) and the FRAZEE model with Hopkins $6^{\circ}$ optics (having the outer diameter of $8 \mathrm{~mm}$ and $5 \mathrm{~mm}$ working channel). The main advantage of the second one was the expanded toolkit. The intracranial access led through the burr hole marked by the navigation system and set in one line with the surgical target. When planning the access, eloquent areas of the brain were saved and the trajectory through the wider lateral ventricle was preferred. After dura incision and arachnoid coagulation the lateral ventricle was punctured according to the planned trajectory and the ventricular system was cannulated. Approximately $10 \mathrm{ml}$ of Ringer's solution was administered by a drain placed into the ventricle to broaden its diameter. At the same time, basic hemodynamic parameters (heart rate and blood pressure) were carefully monitored to prevent any increase of the intracranial pressure. Subsequently using the same trajectory the endoscopic sheath with an obturator was introduced, which was replaced in the next stage with the neuroendoscope. During the entire surgery, the operating field was 
rinsed with Ringer's solution, maintaining constant filling of the ventricular system and transparency of the operating field. Once the tumor was located, the neuroendoscope was fixed in the holder. The feasibility of the intended procedure was analyzed first. It was based on the assessment of the tumor in relation to the vessels and surrounding structures. In the case of a lesion having the appearance of a colloid cyst the surface of the tumor was punctured with a needle and its contents were aspirated. Then the entrance point was widened with the grasping forceps, and in the cases of the presence of a solid part it was removed using the biopsy forceps. The
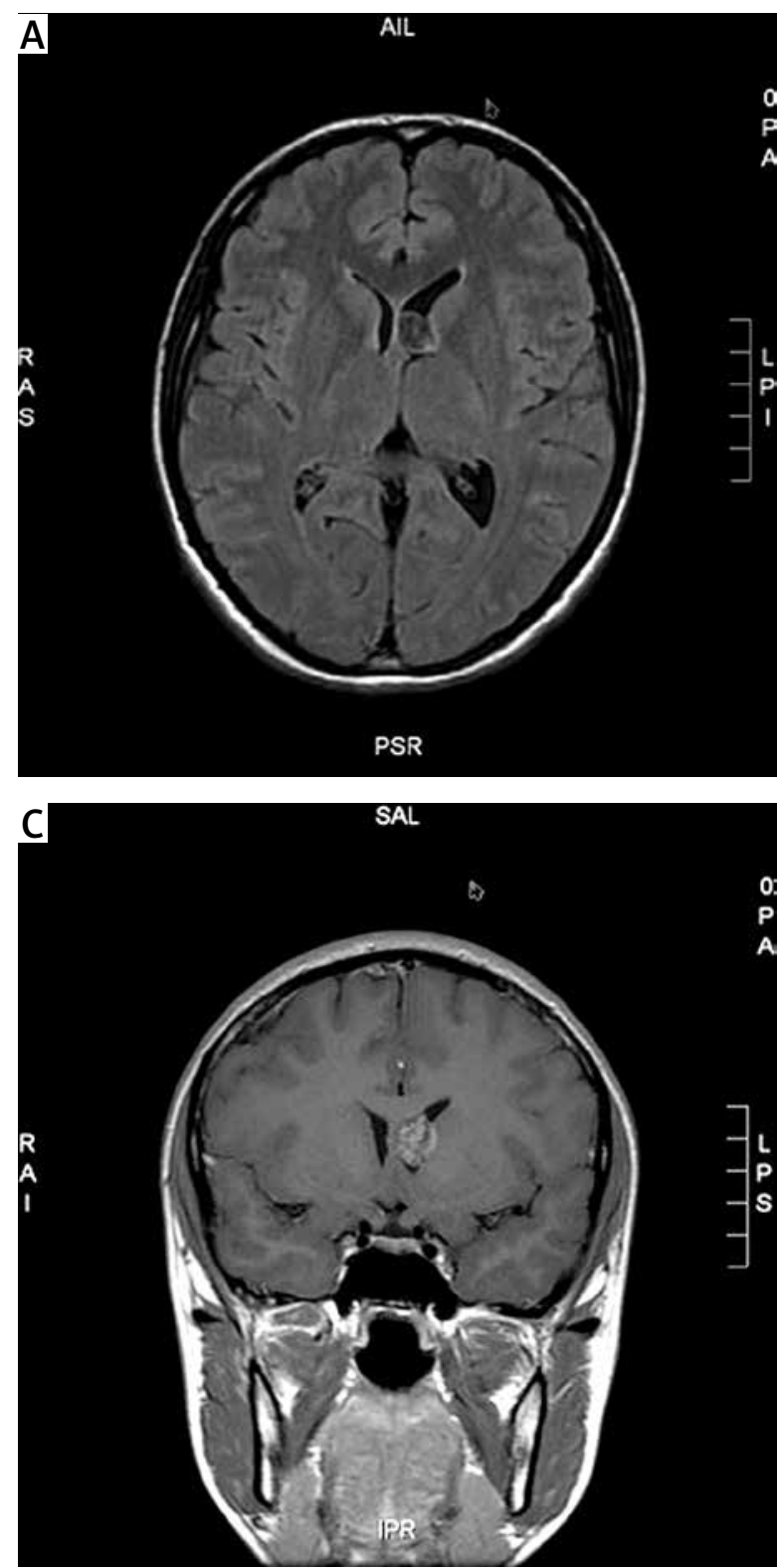

remaining capsule was separated from the choroid plexus using the sharp instrument and bipolar coagulation. Then the capsule was totally removed or it was widely opened, coagulated and left if pushing the total removal carried the risk of bleeding. In solid tumors the operating strategy comprised coagulation of the superficial blood vessels using bipolar coagulation, incision and sampling of the tumor in order to perform the histopathological examination. After confirming the nature of the lesion, there followed tumor reduction by debulking from inside or the total removal of the tumor. During the procedure the tumor's vessels were carefully coagulated, to

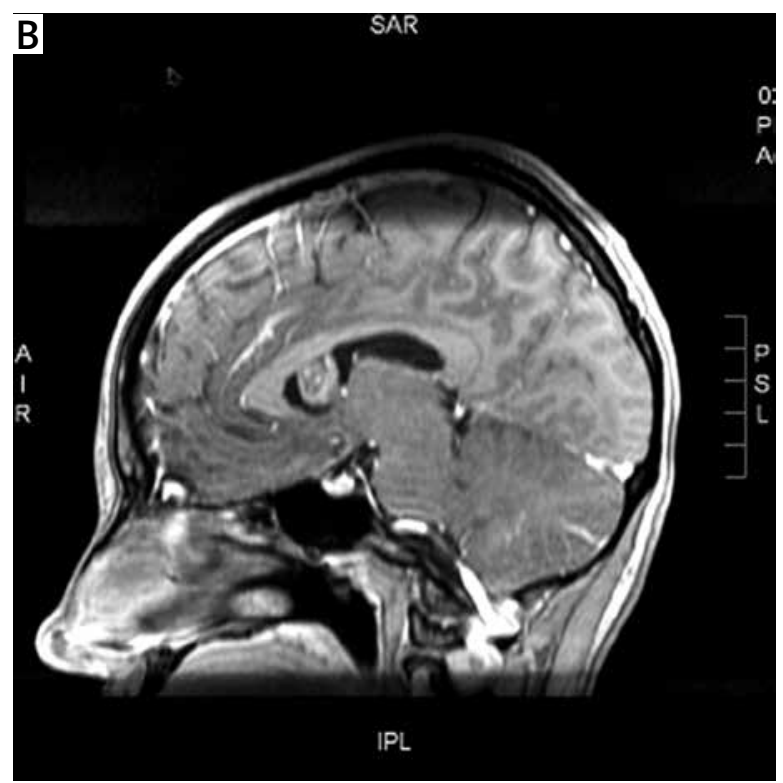

Photo 1. Magnetic resonance imaging (A) axial, (B) sagittal and (C) coronal T1-weighted images: left lateral ventricle tumor adjacent to the interventricular foramen 

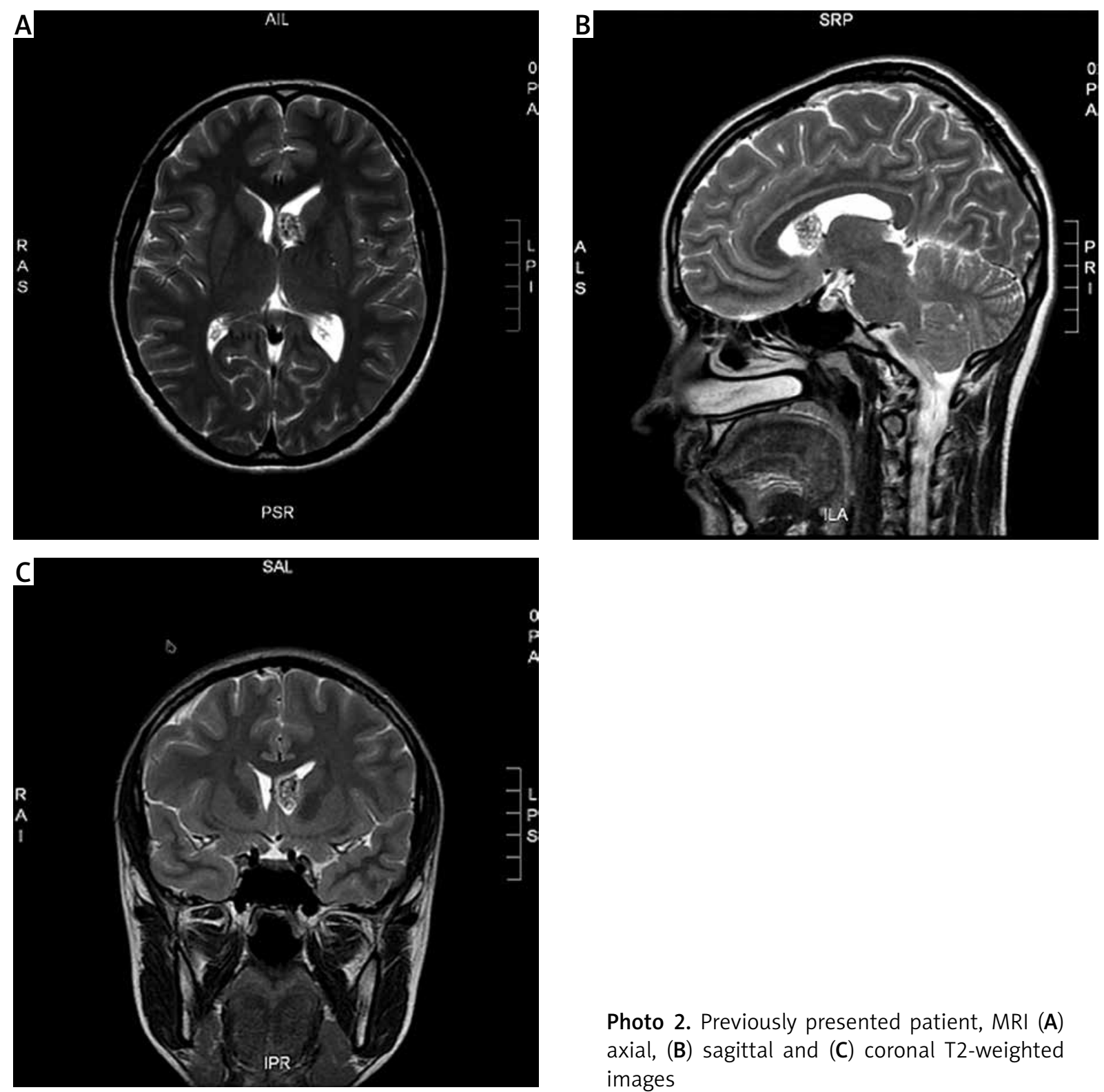

Photo 2. Previously presented patient, MRI (A) axial, (B) sagittal and (C) coronal T2-weighted images

prevent the bleeding that could limit the transparency of the operating field. Deep, uncontrolled penetration of the tumor within the biopsy forceps was avoided. The endoscopic biopsy was performed by taking samples from the parts of tumor presenting sparse vascularization. Efforts were made to collect the tumor samples that had not been the subject of earlier coagulation. In tumors of the posterior part of the third ventricle the biopsy was preceded by endoscopic third ventriculostomy. Hemostasis control and thorough ventricular system irrigation with warm $\left(37^{\circ} \mathrm{C}\right)$ Ringer solution ended the endoscopic part of the procedure. If the full transparency of

cerebrospinal fluid (CSF) was not achieved, a drain was left in the lateral ventricle. After withdrawal of the neuroendoscope the hole in the dura was closed with TachoSil. The galea and the skin were tightly sutured. The ventricular system was rinsed with saline and the drain was removed on the first or second postoperative day (Photos $1 \mathrm{~A}-\mathrm{C}, 2 \mathrm{~A}-\mathrm{C}, 3-5$ ).

Treatment results were evaluated based on the follow-up neurological examinations and on the radiological studies (head CT or MRI) performed directly and in the long-term period after surgery. Patients were subject to long-term outpatient follow-up lasting from 6 to 44 months (mean of 24 months, me- 


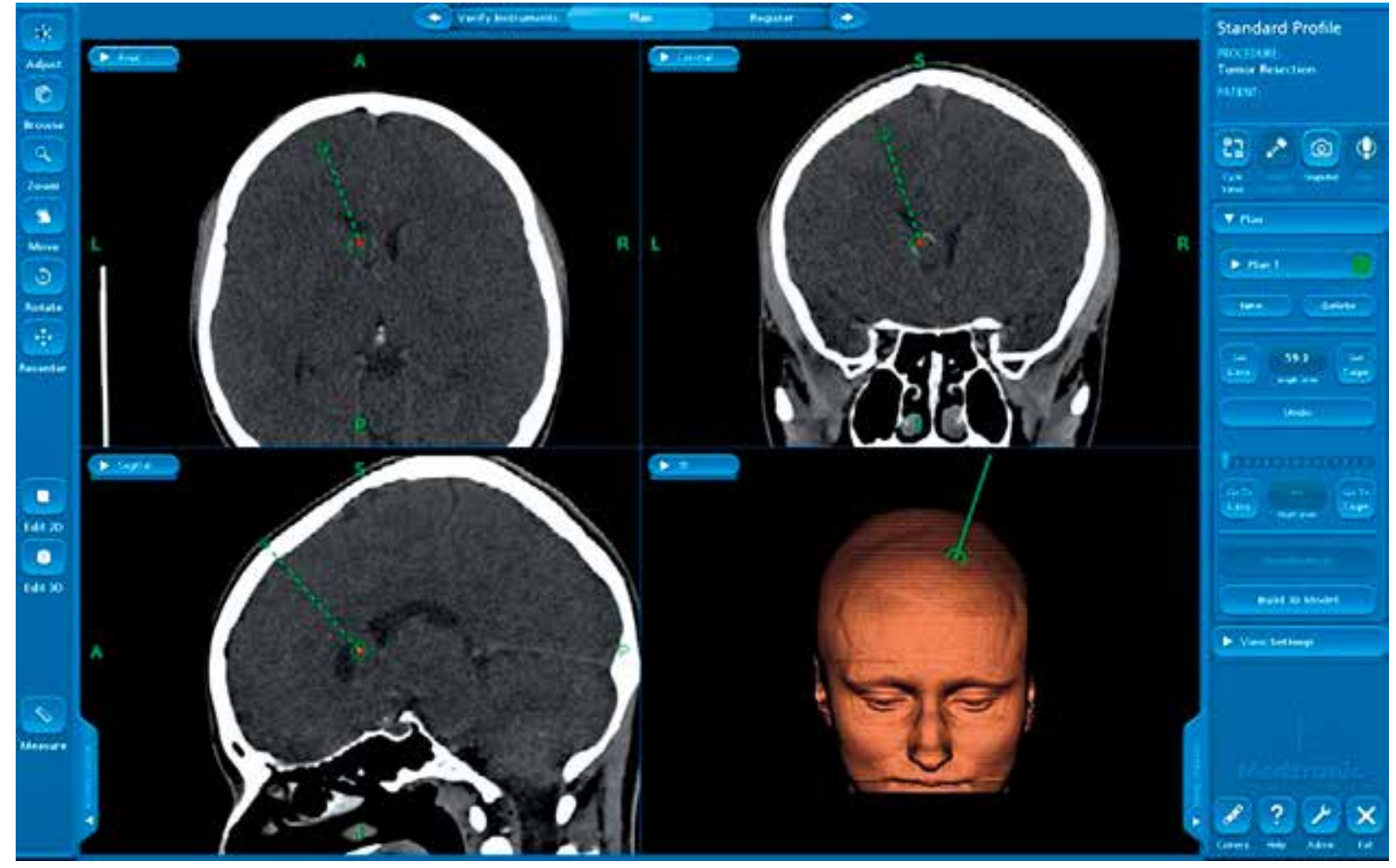

Photo 3. Planning station of neuronavigation system. Planning the optimal trajectory to access the tumor

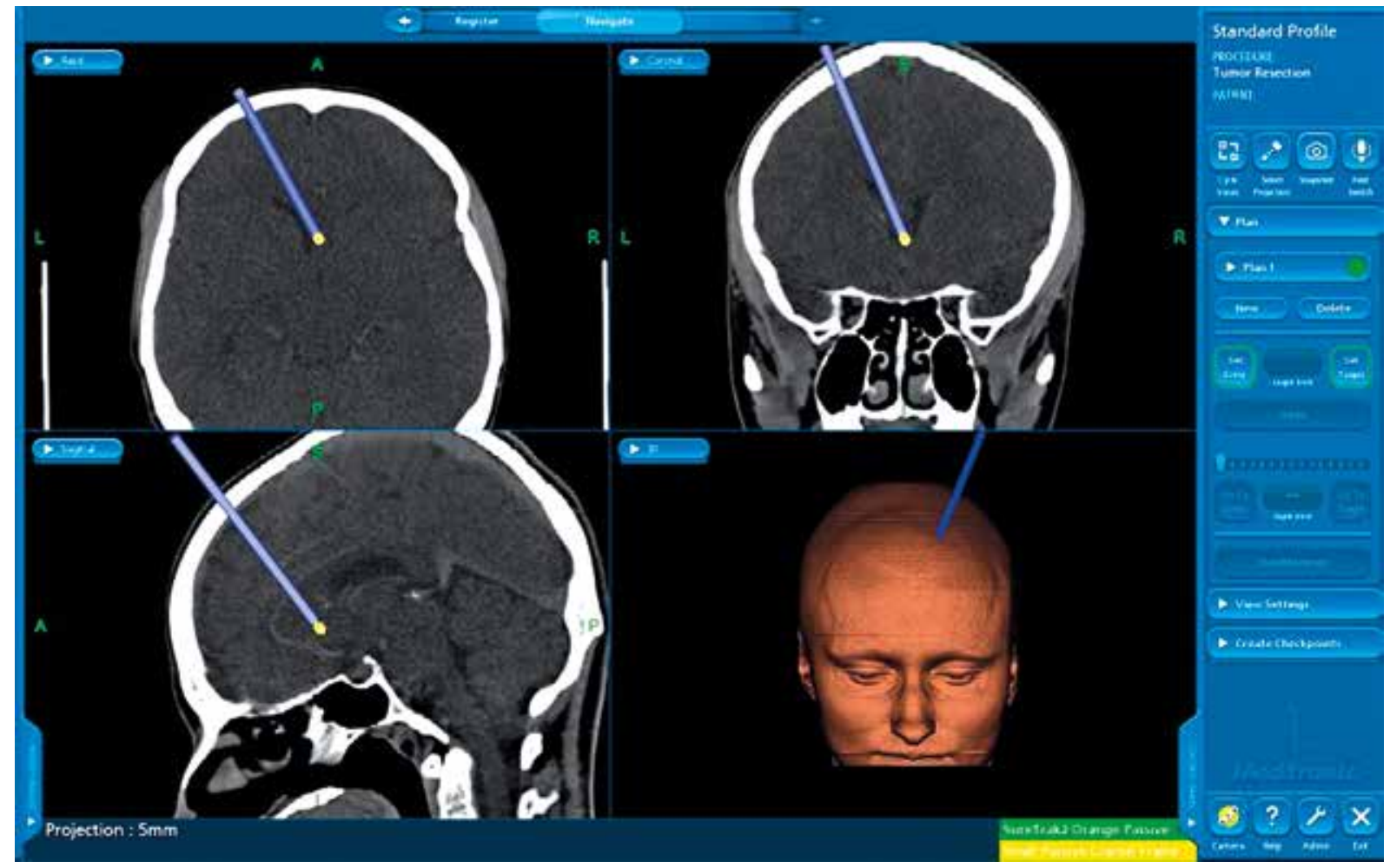

Photo 4. Planning station of neuronavigation system. Image presenting the position of the end of the endoscope 
dian of 23 months). The results were compared to the results obtained in patients with hydrocephalus, who underwent a similar procedure, described in previous papers $[9,10]$.

\section{Results}

The planned aim of surgery was achieved in all patients except one. The colloid cyst of the third ventricle was removed in 5 patients. In 1 patient pilocytic astrocytoma (type of tumor) adjacent to the interventricular foramen was partially resected. In 1 case a glial cyst of the occipital horn of the lateral ventricle was totally removed, and in another case a subependymoma type of tumor in a similar location was partially removed. In 2 cases, a biopsy of the tumor of the posterior part of the third ventricle was carried out to recognize the pineocytoma. The biopsy was abandoned in 1 case due to the high risk of injury to the structures surrounding the interventricular foramen. There were no intraoperative complications or perioperative deaths. One patient developed memory impairments in the direct postoperative period, resulting probably from injury to the fornix; these symptoms disappeared within a few days. We did not observe any symptoms of intracranial hypertension, epilepsy, CSF leakage from the postoperative wound or infectious complications. All patients were discharged from the hospital within 3 days after surgery. The short-term qualitative and quantitative disturbances of consciousness and visual impairments if present before surgery disappeared in the long-term follow-up in all the patients. Headaches resolved in 4 patients. In the remaining patients headaches persisted a little longer, which was associated with the presence of postoperative pneumocephalus. The early and longterm follow-up neuroimaging studies did not reveal any signs of bleeding at the site of surgery, neither hydrocephalus development nor tumor regrowth, during the observation period. The results of treatment in the study group did not differ significantly from those achieved in patients operated on with the enlarged ventricular system, which was the subject of separate studies.

\section{Discussion}

Endoscopic surgery in patients with a narrow ventricular system brings difficulties in cannulation of the ventricular system. The achievement of

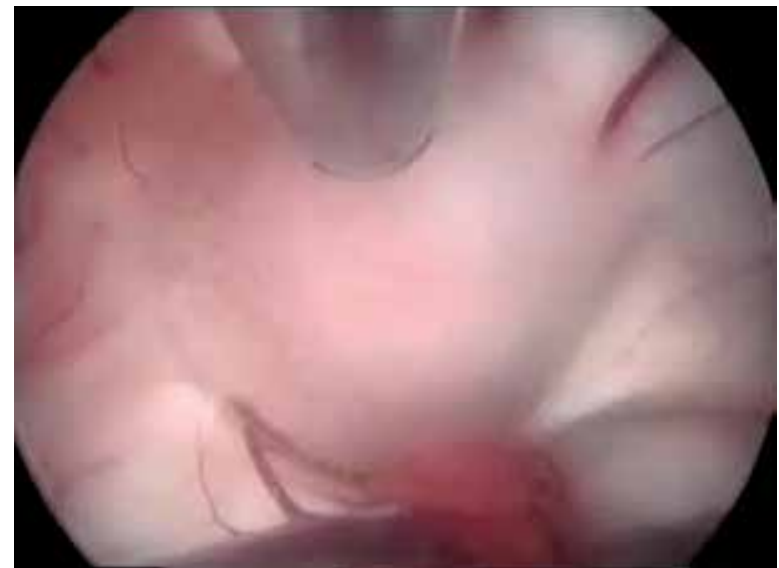

Photo 5 . View by the optics of the endoscope

the operation target entails a lot of risk because of the limited space to maneuver the instruments. In 1997 Yamamoto et al. described the first three cases of patients with the regular width of the ventricular system, who successfully underwent lateral ventricle ventriculoscopy due to arachnoid cysts (2 patients) and subependymal hematoma (1 patient). Procedures were performed using a neurofiberoscope. In conclusion they stated that patients with small intraventricular lesions may benefit from this method of treatment regardless of the ventricle size [6]. In 2005 Harris et al. described the application of stereotactic and intraoperative head CT-assisted endoscopy in resection of intraventricular tumors and colloid cysts. However, their study included patients with an enlarged ventricular system, while the main advantages of the method were minimization of retraction and satisfactory visualization [7]. Gabb and Schroeder believe that patients with a narrow ventricular system pose a serious challenge in endoscopic surgery of tumors, and in these cases precise navigation is helpful to determine the optimal access point and trajectory to the target of surgery [11]. The first stereotactic navigation devices have been more used in patients without hydrocephalus than with hydrocephalus - respectively $73 \%$ to $39 \%$ [4]. In practice, the use of frameless navigation is commonly accepted wherever there is a significant deformity of the anatomic structures, e.g. the slit ventricle syndrome, or if midline shift occurs $[12,13]$.

Using neuronavigation to cannulate the narrow ventricular system and to perform under its control the various intraventricular procedures has many followers [1, 3, 11, 13, 14]. It has undeniable advantag- 
es - the direction of the endoscope's introduction is exactly known, the image is independent of the view obtained through the endoscope optics, and the adjacent anatomical structures are clearly visible. In other words, the advantages of neuronavigation compensate the weak points of neuroendoscopy, which are: possible occurring difficulties in spatial orientation while introducing the endoscope, limited field of view of the endoscope and quite easy loss of visibility even if slight bleeding occurs. A very important element of neuronavigation-assisted endoscopic surgery is to maintain the filling of the ventricular system with fluid at a constant level after the drain has already been introduced to the lateral ventricle. Its excessive loss may cause the displacement of intracranial structures and the phenomenon called brain shift, which results in incompatibility of the navigation image and the real surgical anatomy. The attempts to eliminate this inconvenience are based on the use of intraoperative ultrasonography and low-field MRI [15-17]. The authors avoided the phenomenon of brain shift through the appropriate positioning of the head to prevent excessive CSF leakage. At the same time, the gravity-enforced constant irrigation of the operative field within the Ringer solution complemented the potential CSF leakage while maintaining the transparency of the operating field. It requires control over the opening of the outflow port of the endoscope and the careful cooperation of the anesthesiologist, who should report any changes in the basic hemodynamic parameters (bradycardia, increase in blood pressure) announcing an increase of the intracranial pressure.

Performing endoscopic procedures in patients without hydrocephalus may cause many difficulties. Therefore, they should be performed by neurosurgeons having sufficient experience in neuroendoscopy as well as in utilization of neuronavigation. The final qualification for surgery often remains an open question; however, the best candidates for this method of treatment seem to be patients with tumors situated in the lateral ventricles or in the anterior part of the third ventricle. Access to tumors located in the posterior part of the third ventricle remains problematic and may result in injury of the adjacent anatomical structures. Souweidane, having regard to the risk of injury to the choroid plexus, internal cerebral veins and thalamus, discourages operations in the posterior part of the third ventricle if the ventricle is narrow [4]. In the presented material, one case of forcing the access to the tumor in the mentioned location through the narrow interventricular foramen resulted in temporary symptoms of fornix injury. In the second case, considering the already acquired knowledge, the biopsy was abandoned after intraoperative assessment of the anatomical conditions. The technique of intraventricular tumor resection in patients without hydrocephalus is no different from the cases with hydrocephalus. However, due to the very limited field of operation, multidirectional assessment of the feasibility of the intended procedure is necessary. It should be the result of intraventricular anatomical conditions, tumor morphology with regard to its cohesion and vascularization and to the capacity of the available equipment. Even partial removal of a tumor that is located in the neighborhood of the interventricular foramen may bring distinct benefits to the patient and protect against the development of acute hydrocephalus. Besides colloid cysts, also glial-derived low-grade (WHO I) tumors can be found in this location, which after partial removal do not present any features of regrowth for years. Endoscopic biopsy gives a lot more opportunities compared to conventional biopsy. It allows for inspection of the biopsy site with direct visualization and targeted biopsy with preservation of the choroid plexus or vascular structures. It also enables, if indicated, sampling of the cerebrospinal fluid, third ventriculostomy, septostomy or decompression of cystic lesions.

The results of endoscopic procedures with a narrow ventricular system have not been widely presented in the literature. Similar publications that have discussed the issues of removal or biopsy of tumors in both pediatric and adult populations have reported successful achievement of the surgery objective with a good clinical outcome and no major complications. It is believed that with the navigation system there are no contraindications to perform these procedures in patients without hydrocephalus [4, 5, 18]. These findings are consistent with those obtained by the authors and break down the previously existing views on the feasibility and efficiency of endoscopic surgery in the treatment of intraventricular tumors. The discussed method seems to be promising, and its ultimate usefulness will be closely linked to the further development of medical technologies.

\section{Conclusions}

The presence of hydrocephalus is not necessary to perform endoscopic surgery within the ventricular 
system. The resection or biopsy of tumors located in the lateral ventricles or in the anterior part of the third ventricle is feasible. However, in each case it should be preceded by a thorough analysis of the feasibility of the endoscopic procedure and supported with the neuronavigation system.

\section{Acknowledgments}

Publication was supported by the Faculty of Medicine of Jagiellonian University Medical College (Leading National Research Centre 2012-2017).

\section{Conflict of interest}

The authors declare no conflict of interest.

\section{References}

1. Grunert P, Hopf N, Perneczky A. Frame-based and frameless endoscopic procedures in the third ventricle. Sereotact Funct Neurosurg 1997; 68: 80-9.

2. Fiorindi A, Longatti P. A restricted neuroendoscopic approach for pathological diagnosis of intraventricular and paraventricular tumours. Acta Neurochir (Wien) 2008; 150: 1235-39.

3. Roszkowski M. Neuronavigation - image guided surgery, current application in neurosurgery. Probl Lek 2006; 45: 17-26.

4. Souweidane MM. Endoscopic surgery for intraventricular brain tumors in patients without hydrocephalus. Neurosurgery 2005; 57 (4 Suppl.): 312-8.

5. Song JH, Kong DS, Seol HJ, et al. Transventricular biopsy of brain tumor without hydrocephalus using neuroendoscopy with nawigation. J Korean Neurosurg Soc 2010; 47: 415-9.

6. Yamamoto M, Oka K, Takasugi S, et al. Flexible neuroendoscopy for percutaneous treatment of intraventricular lesion in the absence of hydrocephalus. Minim Invasive Neurosurg 1997; 40: 139-43.

7. Harris AE, Hadjipanayis CG, Lunsford LD, et al. Microsurgical removal of intraventricular lesions using endoscopic visualization and stereotactic guidance. Neurosurgery 2005; 56: 125-32.

8. O'Hayon BB, Drake JM, Ossip MG, et al. Frontal and occipital horn ratio: a linear estimate of ventricular size for multiple imaging modalities in pediatric hydrocephalus. Pediatr Neurosurg 1998; 29: 245-9.

9. Stachura K, Libionka W, Czepko R. The use of neuroendoscopy in the treatment of intraventricular or paraventricular brain tumors. Neurol Neurochir Pol 2005; 39: 101-7.

10. Stachura K. The efficiency of neuroendoscopy procedures in the chosen cases of intracranial pathology at adult in the longwave evaluation. Jagiellonian University Press, Krakow 2010.

11. Gaab MR, Schroeder HWS. Neuroendoscopic approach to intraventricular lesions. J Neurosurg 1998; 88: 496-505.

12. Mcinerney J, Roberts DW. Frameless stereotaxy of the brain Mt Sinai J Med 2000; 67: 300-10.

13. Schroeder HWS, Wagner W, Tschiltschke W, et al. Frameless neuronavigation in intracranial endoscopic neurosurgery. J Neurosurg 2001; 94: 172-9.
14. Decq P, Le Guerinel C, Brugieres P, et al. Endoscopic management of colloid cysts. Neurosurgery 1998; 42: 1288-96.

15. Fahlbusch R, Ganslandt O, Nimsky C. Intraoperative imaging with open magnetic resonance imaging and neuronavigation. Childs Nerv Syst 2000; 16: 829-31.

16. Joedicke A, Deinsberger W, Erbe $H$, et al. Intraoperative trii-dimensional ultrasonography: an approach to register brain shift using multidimensional image processing. Minim Invasive Neurosurg 1998; 41: 13-9.

17. Kanner AA, Vogelbaum MA, Mayberg MR, et al. Intracranial navigation by using low-field intraoperative magnetic resonance imaging: preliminary experience. J Neurosurg 2002; 97: $1115-24$.

18. Lee MH, Kim HR, Seol HJ, et al. Neuroendoscopic biopsy of pediatric brain tumors with small ventricle. Childs Nerv Syst 2014; 30: 1055-60.

Received: 12.01 .2016 , accepted: 12.06 .2016 